\title{
Autoimmune Pancreatitis: An Autoimmune or Immunoinflammatory Disease?
}

\author{
Yvonne Hsieh, Sudhanshu Agrawal, Leman Yel and Sudhir Gupta*
}

Division of Basic and Clinical Immunology, University of California, Irvine, CA 92697, USA

\begin{abstract}
Autoimmune pancreatitis (AIP) has been widely presumed to be an autoimmune disease that is characterized by elevated IgG and/or IgG4, the presence of autoantibodies, and an infiltration of lymphocytes and plasma cells with fibrosis. However, no detailed immunological studies have been published. To define immunological changes in AIP in detail, and to review evidence for autoimmunity which may be antigen specific and may play a role in the pathogenesis of AIP, and therefore, to determine whether AIP is an autoimmune disease. A detailed immunological investigation for both innate and adaptive immune responses was performed in a patient with AIP. Review of literature was performed from Pub med, and Medline search. Immunological analysis of patient with AIP revealed increased production of proinflammatory IL-6, and IL-17, and increased NK cell activity. No organ-specific or non-specific antibodies were detected. There was no correlation between serum IgG4 with disease activity or response to steroid therapy. Review of literature revealed lack of auto-antigen-specific $\mathrm{T}$ and $\mathrm{B}$ cell responses in AIP, and autoantibodies are present only in a subset of patients, and are not specific to pancreatic tissue antigens. Therefore, we propose the term Immunoinflammatory pancreatitis rather than an autoimmune pancreatitis.
\end{abstract}

Keywords: NK cells, IL-6, IL-17, autoantibodies, IgG4.

\section{INTRODUCTION}

The term "autoimmune pancreatitis" (AIP) or "autoimmune-related pancreatitis" was first introduced by Yoshida et al. in 1995 to describe a form of chronic pancreatitis that is associated with autoimmune manifestations [1]. This was based upon pancreatitis in a 68-year-old woman associated with the presence of hypergammaglobulinemia, systemic autoantibodies (antinuclear antibody, anti-thyroglobulin antibody, and anti-microsomal antibody), and response to steroid therapy. Since then, numerous cases have been reported with similar findings in relation to the disease response to steroid therapy and elevated $\mathrm{IgG} 4$. However, serological data have been inconsistent, especially for the presence of autoantibodies, and the etiopathogenesis of the disease remains elusive.

Autoimmune pancreatitis is typically characterized by a diffuse or segmental narrowing of the main pancreatic duct on imaging, elevated $\mathrm{IgG}$ and/or IgG4, the presence of autoantibodies, an infiltration of lymphocytes and plasma cells, and presence of fibrosis. The autoantibodies that have been examined include ANA, anti-microsomal antibodies, anti-thyroglobulin antibodies, and antibodies against pancreatic secretory trypsin inhibitor, lactoferrin, and carbonic anhydrase [2-5]. However, the presence of these autoantibodies has been only sporadically documented in limited numbers of cases. In addition, many of these autoantibodies are directed against antigens that are also present in the salivary gland, biliary duct, and distal renal tubules. The detection of $\mathrm{IgG}$, IgG4, antinuclear antibodies, and rheumatoid factor

*Address correspondence to this author at the Division of Basic and Clinical Immunology Medical Sciences I, C240 University of California, Irvine Irvine, CA 92697-4069, USA; Tel: 949-824-5818; Fax: 949-824-4362;

E-mail: sgupta@uci.edu collectively has been suggested to increase the sensitivity of detection of the disease [6]. However, there is no current disease-specific antibody that has been identified [7].

The diagnostic difficulties have also been complicated by the different sets of criteria that have been proposed by authors from numerous countries, which make it difficult to compare the data. The Japanese criteria include imaging, laboratory, and histopathological criteria [8]. The Korean criteria include imaging, laboratory, histopathological, involvement of other organs, and response to steroid therapy [9]. The Mayo criteria include histology, imaging, serology including elevated serum IgG4 levels, other organ involvement, and response to steroids [10, 11]. Recently, Japanese and Korean group of investigators joined to produce the Asian diagnostic criteria, which included imaging, serology (high levels of serum IgG or IgG4, detection of autoantibodies), and histopathology with an optional criterion of response to steroid therapy [12].

Despite the fact that this disease has been categorized as autoimmune, there has not been antigen-specific autoantibodies or pancreatic antigen-specific $\mathrm{T}$ cells that are consistently or exclusively present in patients with AIP. Here we present an extensive immunological analysis in a patient with AIP, and a review of the literature.

The patient is a 70-year old Chinese male who was evaluated for persistent abdominal pain and weight loss. His physical examination was unremarkable except for an enlarged submandibular gland. The medical history was remarkable for only hypertension and benign prostatic hyperplasia, with no history of alcohol, tobacco, or recreational drug use. His family history was significant for a brother with type 2 diabetes mellitus and a sister who died from complications of rheumatoid arthritis. The endoscopic ultra- 
sound (EUS) showed diffuse lobularity of the pancreas with narrowing of the pancreatic duct, findings consistent with "autoimmune pancreatitis". Because of the presence of a large submandibular gland, a PET scan was performed, which showed activity in the prostate, pancreas, lung, and left submandibular lymph nodes. The patient refused a pancreatic biopsy but did agree to undergo a core needle biopsy of the submandibular lymph node, which showed many small round lymphocytes and plasma cells infiltrating between ducts and acinar cells. Flow cytometry of the lymphocytes revealed mostly T cells $(70 \%)$ and a few B cells $(30 \%)$. Patient was started on prednisone $40 \mathrm{mg}$ daily. His symptoms improved while on steroids and experienced a marked improvement in his EUS findings. As the steroids were initially tapered, he developed an increase in the size of his submandibular mass, a mild rise in IgG4, as well as an increase in his fatigue and weight loss. However, throughout the disease course, his IgG4 did not correlate with symptoms or response to prednisone (Fig. 1). The patient eventually developed diabetes, requiring insulin therapy to control blood sugar levels.

\section{METHODS}

This study was approved by the Institutional Review Board (Human) of the University of California, Irvine. A written consent was obtained.

\section{Immunological Evaluation}

Quantitative IgM, IgG, IgA, and IgE levels were measured by nephelometric method. T cells, $\mathrm{T}$ cell subsets, B cells, and natural killer (NK cells) cells were analyzed by multicolor flow cytometry using direct fluorochromeconjugated antibodies against CD3, CD4, CD8, CD19, CD16, and CD56, and isotype controls. The lymphocyte proliferation responses to mitogens (phytohemagglutinin, concanavalin A, pokeweed mitogen) and antigens (purified protein derivative, mumps antigen, tetanus toxoid, Candida albicans) were assessed by DNA synthesis.

\section{Cytokine Production}

\section{IL-6, IL-10, TNF- $\alpha$, IFN- $\gamma$ IL-17}

$1 \times 10^{6}$ peripheral blood mononuclear cells $/ \mathrm{ml}$ were activated with recombinant anti-CD3 $(1 \mathrm{mg} / \mathrm{ml})$ and CD28 $(1 \mathrm{mg} / \mathrm{ml})$ for 2 days and supernatants were collected. IL-6, IL-10, TNF- $\alpha$, and IFN- $\gamma$ were assayed by ELISA (BD Pharmingen, San Diego, CA). For IL-17, Nunc MaxiSorp plates were coated with $1 \mathrm{mg} / \mathrm{ml}$ of purified IL-17 (BD Biosciences) overnight at $4^{\circ} \mathrm{C}$. After overnight incubation, the plates were blocked with phosphate-buffered saline containing $10 \%$ fetal bovine serum, washed and incubated with suitable dilutions of the supernatants. Bound IL-17 was detected using biotinylated detection antibodies $(1 \mathrm{mg} / \mathrm{ml})$ and HRPconjugated streptavidin. After washing and addition of substrate, the optical density in the wells was measured at $450 \mathrm{~nm}$ and background values were subtracted.

\section{FOXP3+ Treg}

Peripheral blood mononuclear cells were stained with CD4+ PerCP and CD25 FITC, and fixed with 2\% paraformaldehyde. Cells were centrifuged and fixative was removed. Cells were permeabilized with wash buffer and antiFOXP3 antibody was added for 30 minutes. Appropriate isotype control was used. The cells were acquired by BD FACSCalibur and the data was analyzed by FlowJo (Treestar software, Ashland,OR).

\section{Natural Killer Cell Activity}

Four microliters of carboxyfluorescein succinimidyl ester (CFSE) dye diluted with PBS was added to K562 cells

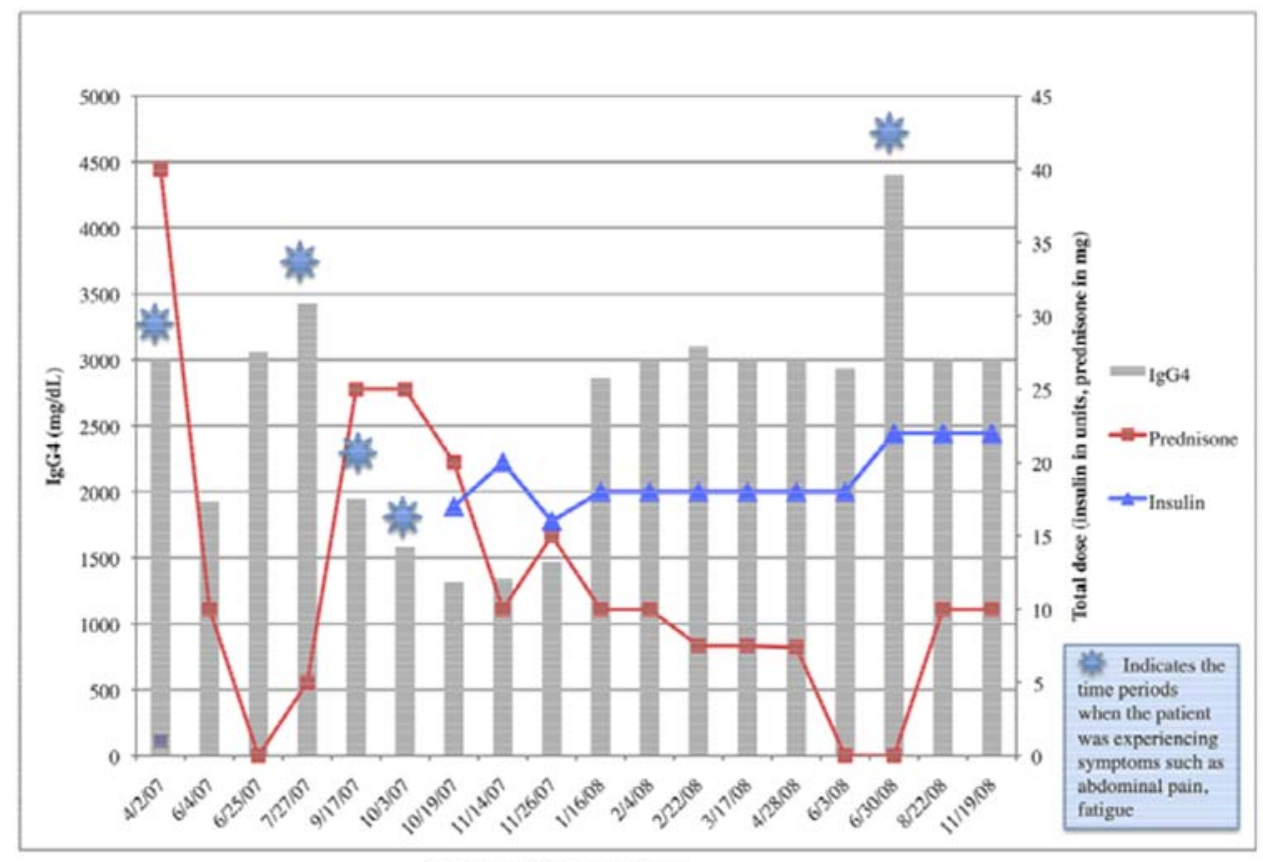

Dates of Observation

Fig. (1). Clinical Course, Response to Corticosteroids, and Serum IgG4 levels in a patient with Autoimmune Pancreatitis. 
Table 1. Immunologic Analysis of a Patient with Autoimmune Pancreatitis

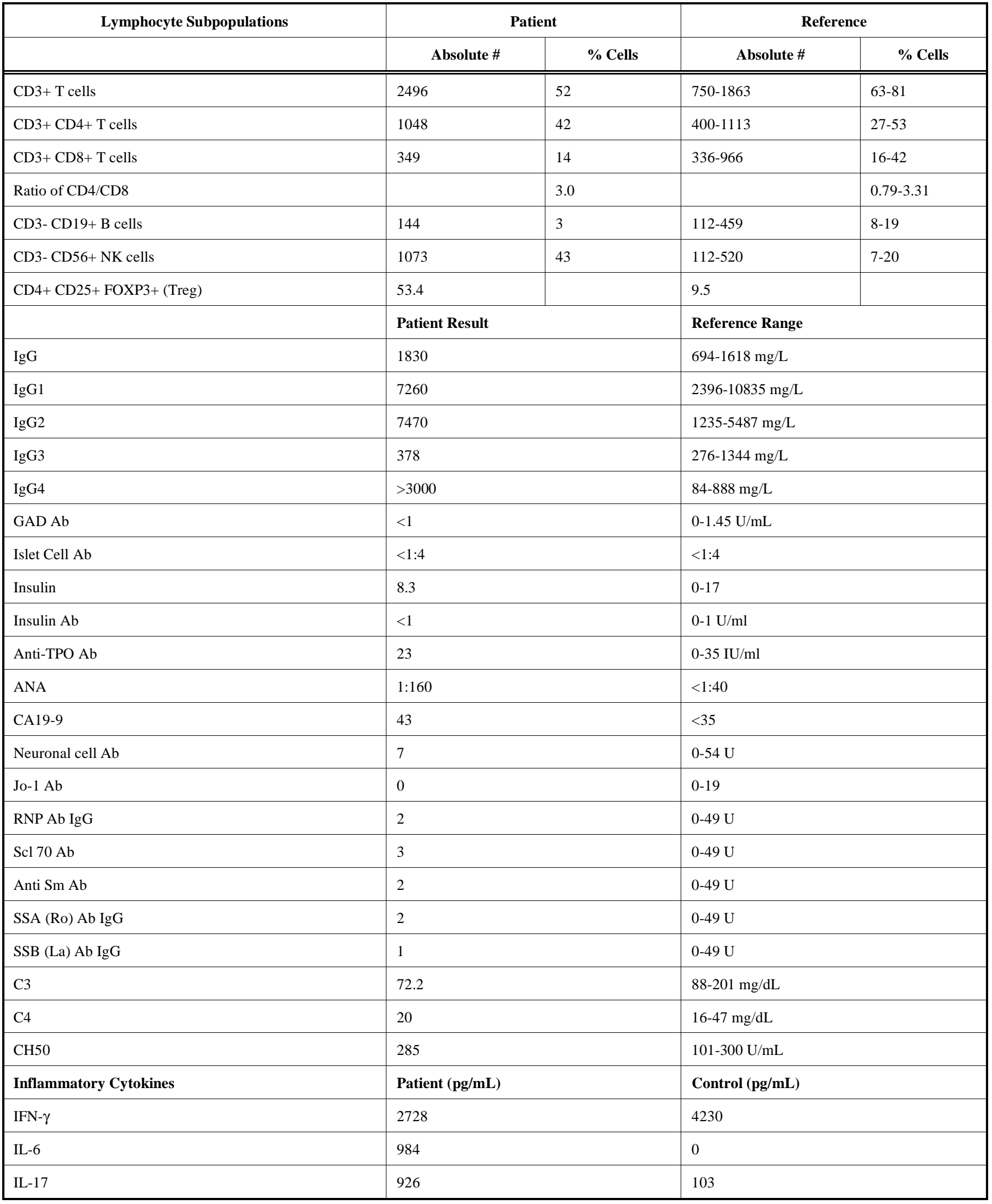


(Table 1). Contd....

\begin{tabular}{|c|c|c|c|c|}
\hline Cytokines & \multicolumn{2}{|c|}{ Patient } & \multicolumn{2}{|r|}{ Reference } \\
\hline IL-10 & \multicolumn{2}{|l|}{158} & \multicolumn{2}{|l|}{160} \\
\hline TNF-a & \multicolumn{2}{|l|}{131} & \multicolumn{2}{|l|}{464} \\
\hline In Vitro Lymphocyte Proliferative Response (cpm) & \multicolumn{2}{|l|}{ Patient } & \multicolumn{2}{|c|}{ Reference Range } \\
\hline Phytohemagglutinin (PHA) & \multicolumn{2}{|l|}{67,328} & \multicolumn{2}{|c|}{$142,821-324,200$} \\
\hline Concanavalin A (Con A) & \multicolumn{2}{|l|}{4,180} & \multicolumn{2}{|c|}{$156,821-341,795$} \\
\hline Pokeweed mitogen (PWM) & \multicolumn{2}{|l|}{22,300} & \multicolumn{2}{|c|}{$23,648-90,931$} \\
\hline Mumps antigen & \multicolumn{2}{|l|}{220} & \multicolumn{2}{|c|}{$2,052-26,495$} \\
\hline Tetanus toxoid & \multicolumn{2}{|l|}{257} & \multicolumn{2}{|c|}{$6,092-94,539$} \\
\hline Candida albicans antigen & \multicolumn{2}{|l|}{93} & \multicolumn{2}{|c|}{$13,249-60,917$} \\
\hline \multicolumn{5}{|l|}{ NK Cell Function Results ( \% cytotoxicity) } \\
\hline Effector:Target Ratios & $12.5: 1$ & $25: 1$ & $50: 1$ & $100: 1$ \\
\hline Patient ( 24 hrs) & 33 & 54 & 70 & 75 \\
\hline Normal range (fresh cells) & $4-19$ & $8-37$ & $16-51$ & $28-64$ \\
\hline \multicolumn{5}{|l|}{ Lytic Units $/ 10^{7}$ Effector Cells } \\
\hline Patient (24 hours) & \multicolumn{4}{|l|}{67} \\
\hline Normal range (fresh cells) & \multicolumn{4}{|l|}{$8-32$} \\
\hline
\end{tabular}

(target cells). The cells were washed twice with RPMI 1640 medium. 1x $10^{5}$ K562 cells were mixed with different concentrations of peripheral blood mononuclear cells to obtain effector:target ratios of 12.5:1, 25:1, 50:1, and 100:1. The mixture was then incubated at $37^{\circ} \mathrm{C}$ for four hours. $5 \mathrm{~mL}$ of 7-amino-actinomycin D (AAD-7) was then added and incubated for another 15 minutes at $37^{\circ} \mathrm{C}$. The cells were analyzed via BD FACSCalibur (Becton Dickinson, San Jose, CA). Lytic unit activity and percentage of NK cell killing were calculated.

\section{RESULTS}

\section{Immunological Findings}

Laboratory evaluation revealed elevated levels of total $\mathrm{IgG}$ of $1830 \mathrm{mg} / \mathrm{L}$ and $\mathrm{IgG} 4$ of $>3000 \mathrm{mg} / \mathrm{L}$ (Table 1). The panel of autoantibodies was negative, except for an elevated anti-nuclear antibody (ANA) at 1:160. Complement levels were normal. The lymphocyte subsets showed a low number of $\mathrm{B}$ and $\mathrm{T}$ cells, but an increased number of natural killer cells. The evaluation of cytokine production revealed an increased level of IL- 6 and IL-17, with low levels of IFN- $\gamma$ and TNF- $\alpha$ compared to control. Natural killer cell cytotoxicity was increased. Responses to mitogens and antigens were impaired. FOXP3+ T regulatory cells were elevated at baseline as compared to control.

\section{Literature Review Data}

Review of literature revealed that only $67 \%$ (54/81) of patients had an elevated IgG4 (Table 2). Only 37\% (46/124) of subjects had a positive ANA, with the definition of a positive ANA varying anywhere from 1:20 to 1:80, depending on the author, and rheumatoid factor was positive in $18 \%$ $(15 / 85)$ of cases. Approximately $10 \%$ (4/42) had a positive anti-smooth muscle antibody, $3 \%(1 / 33)$ had a positive islet cell antibody, $6 \%(2 / 32)$ had a positive glutamic acid decarboxylase antibody, $0 \%(0 / 4)$ had a positive anti-doublestranded DNA antibody, $0 \%(0 / 3)$ had a positive microsomal antibody, 42\% (11/26) had a positive pancreatic secretory trypsin inhibitor antibody, and 50\% (16/32) had a positive carbonic anhydrase antibody. The highest percentage of a positive autoantibody discovered was those of lactoferrin antibody at $68 \%(19 / 28)$.

\section{DISCUSSION}

Unlike many autoimmune diseases, AIP is a rare form of pancreatitis occurring predominantly in males and in the $5^{\text {th }}$ and $6^{\text {th }}$ decade of life. In 1961 Sarles and colleague [13] reported a form of idiopathic chronic pancreatitis suspected to be induced by an autoimmune mechanism. In 1991, Kawaguchi et al., reported 2 cases of an unusual inflammatory disease of the pancreas and biliary tract with histology demonstrating lymphoplasmacytic sclerosing pancreatitis [14]. However, the term AIP was first coined in 1995 by Yoshida and associates [1], who first proposed the concept of AIP characterized by increased levels of $\mathrm{IgG}$ or $\mathrm{IgG} 4$, presence of autoantibodies, diffused irregular narrowing of the main pancreatic duct and enlargement of the pancreas.

Autoimmune pancreatitis is a rare systemic fibrotic inflammatory disorder of the pancreas associated with unique clinical and histopathological characteristics, family history of autoimmune diseases, presence of autoantibodies, presence of IgG4+ cells in duodenal and pancreatic biopsy tis- 
Table 2. Autoantibodies in Autoimmune Pancreatitis

\begin{tabular}{|l|l|l|l|}
\hline \multicolumn{1}{|c|}{ Marker } & \multicolumn{1}{|c|}{$\#$} & \multicolumn{1}{|c|}{ Reference } \\
\hline \hline IgG4 & $54 / 81$ & $67 \%$ & {$[29-32,36,38,39]$} \\
\hline ANA & $46 / 124$ & $37 \%$ & {$[5,29-31,35,38,39]$} \\
\hline CA 19-9 & $4 / 17$ & $24 \%$ & {$[29,35,37]$} \\
\hline Rheumatoid Factor & $15 / 85$ & $18 \%$ & {$[5,30,31,37,38,39]$} \\
\hline Anti smooth muscle Ab* & $4 / 42$ & $10 \%$ & {$[5,31,35,37]$} \\
\hline Anti-islet cell Ab & $1 / 33$ & $3 \%$ & {$[5,33-37]$} \\
\hline Anti-glutamic acid decarboxylase Ab & $2 / 32$ & $6 \%$ & {$[5,33,35,36,37]$} \\
\hline Anti-microsomal Ab & $0 / 3$ & $0 \%$ & {$[37,38]$} \\
\hline Anti-pancreatic secretory trypsin inhibitor Ab & $11 / 26$ & $42 \%$ & {$[5]$} \\
\hline Anti-carbonic anhydrase Ab & $16 / 32$ & $50 \%$ & {$[5,35,37,39]$} \\
\hline Anti-lactoferrin Ab & $19 / 28$ & $68 \%$ & {$[5,39]$} \\
\hline Anti-mitochondrial Ab & $0 / 43$ & $0 \%$ & {$[5,31,35,39]$} \\
\hline Anti double-stranded DNA & $0 / 4$ & $0 \%$ & {$[36-38]$} \\
\hline$*$ Ab= antibodies. & & & \\
\hline
\end{tabular}

sues, elevated serum $\operatorname{IgG} 4$, and response to corticosteroids [15-21]. The fibrotic inflammatory process can also affect organs such as the bile ducts, salivary glands, and retroperitoneal lymph nodes.

Recently, AIP has been divided on the basis of histological features into two subgroups; type I AIP or lymphoplasmacytoid sclerosing pancreatitis (LPSP), and type II AIP or idiopathic ductal centric pancreatitis (IDCP) [22]. Type I and type II AIP appear to be clinicopathologically, regionally, and ethnically different diseases [23]. In addition to clinicopathological differences, type I AIP is associated with frequently elevated IgG4 levels, predominantly in elderly males, and has a high relapse rate, whereas type II AIP has no gender preferences, is usually found in relatively younger subjects, rarely associated with elevated serum IgG4, and does not relapse. Whether these two types of pancreatitis represent disorders with different pathogenesis, remains unclear. Present criteria for the diagnosis of AIP are geared for type I AIP.

Since its clinical description, there has been a search for a potential serological marker for AIP. A large number of autoantibodies, both organ-nonspecific including ANA, rheumatoid factor (RF), anti-smooth muscle (ASM), and organ-specific autoantibodies, including antibodies against carbonic anhydrase, lactoferrin, pancreatic secretory trypsin inhibitor [2-5] have been reported. However, these organspecific autoantibodies are also present in other disorders, including pancreatic carcinoma. Endo et al., [24] reported amylase a -2A antibodies as a novel diagnostic marker for both AIP and fulminant Type I diabetes since it is elevated in these patients and not in controls, as well as the fact that the titers decrease with steroid treatment. Recently, Frulloni et al., [25] detected peptide $\mathrm{AIP}_{1-7}$, a peptide that has homology with plasminogen-binding protein (PBP) of $H$. pylori, and with an enzyme highly expressed in acinar cells of the pancreas, the ubiquitin-protein ligase $\mathrm{E}$ component nrecognin 2. Antibodies to PBP were present in the majority of patients with AIP; however, they were also present in $10 \%$ of cases with pancreatic cancer. Therefore, this antibody neither could be used as a diagnostic marker nor can it be considered to play a major pathogenic role in AIP. Lohr et al., [26] using gene and protein expression profile and immunoassays, identified trypsinogen as a possible target of the inflammatory process. They reported severe downregulation of pancreatic proteases, both at the RNA and protein level, especially trypsinogen, and detected high titers of antitrypsinogen antibodies. However, their data showed that high titers of these antibodies were also present in non-autoimmune pancreatitis and healthy controls. Furthermore, no difference was observed in the serum level of trypsinogen among these groups. Therefore, this autoantibody cannot be considered a reliable diagnostic marker, or to play a significant role in the pathogenesis of AIP.

Asada et al., [27] analyzed humoral immune responses in experimental AIP in mice. Various autoantibodies directed against autoantigens, including carbonic anhydrase II and lactoferrin, were detected, but none against glutamic acid decarboxylase. Autoantibodies directed against the pancreatic secretory trypsin inhibitor (PSTI) were more prevalent $(91.7 \%)$ than those against carbonic anhydrase II $(33.3 \%)$ or lactoferrin $(45.8 \%)$. Antibodies were directed against the epitope of PSTI that is active in the suppression of trypsin activity. Authors concluded that the autoimmune response to PSTI protein may induce a failure of PSTI activity, resulting in the activation of trypsinogen and the subsequent disease progression.

Prior to these new reported findings, we reviewed the literature to determine the type and frequency of autoantibodies that have been previously examined, by collecting data from a large number of studies to determine the per- 
centage of positive autoantibodies reported in diagnosed cases of AIP. Based on the literature search, the majority of autoantibodies that were analyzed in AIP patients were found to be negative and those positive were nonspecific for the pancreatic antigens, such ANA and RF. The presence of lactoferrin antibody, and elevated IgG4 (67\%) were the most consistent laboratory findings in AIP. Lactoferrin, although it is present in the pancreas, is notably produced by the salivary glands, biliary duct, and renal tubules.

The literature review was supported by our patient's immunological evaluation, in which an extensive panel of autoantibodies was negative, except for an elevated ANA. There were signs of inflammation as evident by elevated levels of proinflammatory cytokines IL-6 and IL-17. T cell deficiency was also observed as suggested by impaired responses to mitogens and recall antigens. The numbers of $\mathrm{CD} 4+\mathrm{CD} 25+\mathrm{FOXP} 3+\mathrm{T}$ regulatory cells $\mathrm{T}$ cells were increased; however, we did not study their functions. Koyabu et al., [28], also observed increased proportions of CD4+ $\mathrm{CD} 25$ high cells, which they assigned to $\mathrm{T}$ regulatory $\mathrm{T}$ cells (not all CD4+CD25high cells are FoxP3 Treg cells). Similarly increased proportions of CD4+CD25+FoxP3+ Treg cells have been observed in the biopsy of patients with AIP. These authors, based upon the presence of IgG4+ plasma cells in these biopsies, suggested that increased Treg cells are playing a role in increased IgG4. However, there is no evidence that IgG4 has any specific autoantibody activity against pancreatic antigens in AIP, or they play any pathogenic role in AIP. It is also unclear whether these Treg cells are functionally active. Furthermore, it is possible that increased Treg cells are compensatory negative regulators for autoreactivity. In our patient we also observed increased numbers and activity of NK cells. Whether increased NK cell activity plays a direct or indirect, via secretion of cytokines, pathogenic role in pancreatitis remains to be defined. Our patient refused the pancreatic biopsy; however, submandibular biopsy did show evidence of the plasma cells and lymphocytes infiltration, which is consistent with the biopsy findings described in AIP. Our patient also had elevated levels of IgG4; however, IgG levels did not correlate with the disease activity, response to steroid therapy, or insulin requirements to control elevated blood glucose levels. Therefore, elevated IgG4 may serve as a marker for the diagnosis of AIP; however, it is unlikely to play a role in the pathogenesis of the disease, or even serve as a reliable marker for the response to therapy or for disease activity.

The clinical findings of AIP may better be explained by the presence of immune dysregulation in the inflammatory pathway leading to inappropriate activation of a variety of inflammatory cytokines, rather than autoimmunity. This is supported by the fact that there has been a lack of presence of pancreas-specific antibodies in the majority of patients with AIP, and the presence of these antibodies in pancreatic cancer as well. The presence of systemic autoantibodies may instead be more of a reflection of the immune system dysregulation. Therefore, a potential role of autoantibodies in the pathogenesis of AIP remains unclear. A role of T cells in the pathogenesis of AIP needs to be investigated in detail.

Based upon histopathology of pancreas reported in the literature, and an extensive immunological analysis of the present case, which show increased production of proinflammatory cytokines IL-6, and IL-17, increased NK cell activity, lack of large number of organ-specific and nonspecific antibodies with the exception of ANA, and lack of correlation of $\mathrm{IgG} 4$ levels with disease activity or response to steroid therapy, we propose that the term AIP be replaced by "immunoinflammatory pancreatitis".

In summary, though the initial reported case of AIP had the presence of elevated gammaglobulin, multiple positive nonspecific autoantibodies, response to steroids, and the presence of Sjogren's syndrome, subsequent cases have not uniformly found these similar features. Many cases do not have the presence of other autoimmune diseases and we found that the majority of patients often do not have consistently positive serologic autoantibodies. A definitive pancreatic tissue antigen-specific antibody that contributes to the pathogenesis of the disease has yet to be identified, which may reflect the possibility that autoimmunity may not be the underlying cause of the disease. The response to steroids, elevated inflammatory cytokines, and lack of a definitive pancreatic tissue specific autoantibody, may be more appropriately seen as an evidence of immune inflammation, and be better described as an "immunoinflammatory pancreatitis".

\section{ACKNOWLEDGEMENT}

None declared.

\section{CONFLICT OF INTEREST}

None declared.

\section{REFERENCES}

[1] Yoshida K, Toki F, Takeuchi T, Watananbe S, Shiratori K, Havashi N. Chronic pancreatitis caused by an autoimmune abnormality. Proposal of the concept of autoimmune pancreatitis. Dig Dis Sci 1995; 40: 1561-8.

[2] Okazaki K, Uchida K, Ohana M, et al. Autoimmune-related pancreatitis is associated with autoantibodies and a Th1/Th2-type cellular immune response. Gastroenterology 2000; 118: 573-81.

[3] Kino-Ohsaki J, Nishimori I, Morita M, et al. Serum antibodies to carbonic anhydrase I and II in patients with idiopathic chronic pancreatitis and Sjogren's syndrome. Gastroenterology 1996; 110: 1579-86.

[4] Frulloni L, Bovo P, Brunelli S, et al. Elevated serum levels of antibodies to carbonic anhydrase I and II in patients with chronic pancreatitis. Pancreas 2000; 20: 382-8.

[5] Asada M, Nishio A, Uchida K, et al. Identification of a novel autoantibody against pancreatic secretory trypsin inhibitor in patients with autoimmune pancreatitis. Pancreas 2006; 33: 20-6.

[6] Kawa S, Hamano H. Serological markers for the diagnosis of autoimmune pancreatitis. J Jpn Pancreas Soc 2007; 22: 641-5.

[7] Okazaki K, Uchida K, Fukui T. Recent advances in autoimmune pancreatitis: concept, diagnosis, and pathogenesis. J Gastroenterol 2008; 43: 409-18.

[8] Okazaki K, Kawa S, Kamisawa T, et al. Research Committee of Intractable Diseases of the Pancreas. Clinical diagnostic criteria of autoimmune pancreatitis: revised proposal. J Gastroenterol 2006; 41: 626-31.

[9] Kim MH, Lee TY. Diagnostic criteria for autoimmune pancreatitis: a proposal of revised Kim criteria. J Gastroenterol Hepatol 2007; 22: A104.

[10] Chari ST. Diagnosis of AIP using its five cardinal features: introducing the Mayo Clinic's HISORt criteria. J Gastroenterol 2007; 42(Suppl 18): 39-41.

[11] Chari ST, Smyrk TC, Levy MJ, et al. Diagnosis of autoimmune pancreatitis: the Mayo Clinic experience. Clin Gastroenterol Hepatol 2006; 4: 1010-6. 
[12] Otsuki M, Chugn JB, Okazaki K, et al. Asian diagnostic criteria for autoimmune pancreatitis: consensus of the Japan-Korea Symposium on Autoimmune Pancreatitis. J Gastroenterol 2008; 43: 403-8.

[13] Sarles H, Sarles JC, Muratore R, Guien C. Chronic inflammatory sclerosis of the pancreas-an autonomous pancreatic disease? Am J Dig Dis 1961; 6: 688-98.

[14] Kawaguchi K, Koike M, Tsuruta K, Okamoto A, Tabata I, Fujita N. Lymphoplasmacytic sclerosing pancreatitis with cholangitis: a variant of primary sclerosing cholangitis extensively involving pancreas. Hum Pathol 1991; 22: 387-95.

[15] Detlefsen S, Drewes AM. Autoimmune pancreatitis. Scand J Immunol 2009; 44: 1397-407.

[16] Zhang L, Smyrk TC. Autoimmune pancreatitis and IgG4-related systemic diseases. Int J Clin Pathol 2010; 3: 491-504.

[17] Pickartz T, Mayerle J, Lerch MM. Autoimmune pancreatitis. Nat Clin Pract Gastroenterol Hepatol 2007; 4: 314-423.

[18] Hamano H, Kawa S, Horiuchi A, et al. High serum IgG4 concentrations in patients with sclerosing pancreatitis. N Engl J Med 2001; 344: 732-8.

[19] Moon SH, Kim MH, Park DH, et al. IgG4 immunostaining of duodenal papillary biopsy specimens may be useful for supporting a diagnosis of autoimmune pancreatitis. Gastrointest Endosc 2010; 71: 960-6

[20] Pannala R, Chari ST. Autoimmune pancreatitis. Curr Opin Gastroenterol 2008; 24: 591-6.

[21] Pezzilli R, Morselli-Labate AM. The concept of autoimmune pancreatitis and its immunological backgrounds. Expert Rev Clin Immunol 2010; 6: 125-36.

[22] Sah RP, Chari SR, Pannala R, et al. Differences in clinical profile and relapse rate of type I versus type II autoimmune pancreatitis. Gastroenterology 2010; 139: 140-8.

[23] Kamisawa T, Notohara K, Shimosegawa T. Two clinicopathological subtypes of autoimmune pancreatitis: LPSP and IDCP. Gastroenterology 2010; 139: 22-5.

[24] Endo T, Takizawa S, Tanaka S, et al. Amylase a-2A autoantibodies: novel marker of autoimmune pancreatitis and fulminant type 1 diabetes. Diabetes 2009; 58: 732-7.

[25] Frulloni L, Lunardi C, Simone R, et al. Identification of a novel antibody associated with autoimmune pancreatitis. N Engl J Med 2009; 361: 2135-42.

[26] Löhr JM, Faissner R, Koczan D, et al. Autoantibodies against the exocrine pancreas in autoimmune pancreatitis: gene and protein expression profiling and immunoassays identify pancreatic enzymes as a major target of the inflammatory process. Am J Gastroenterol 2010; 105: 2060-71.
[27] Asada M, Nishio A, Akamatsu T, et al. Analysis of humoral immune response in experimental autoimmune pancreatitis in mice. Pancreas 2010; 39: 224-31.

[28] Koyabu M, Uchida K, Miyoshi H, et al. Analysis of regulatory T cells and IgG4-plasma cells among patients of IgG4-related sclerosing cholangitis and autoimmune liver diseases. J Gastroenterol 2010; 45: 732-41.

[29] Raina A, Yadav D, Krasinskas A, et al. Evaluation and management of autoimmune pancreatitis: experience at a large US center. Am J Gastroenterol 2009; 104: 2295-306.

[30] Ryu J, Chung J, Park S, et al. Review of 67 patients with autoimmune pancreatitis in Korea. Pancreas 2008; 37: 377-85.

[31] Church N, Pereira S, Deheragoda M, et al. Autoimmune pancreatitis: clinical and radiological features and objective response to steroid therapy in a UK series. Am J Gastroenterol 2007; 102: 241725 .

[32] Nishino T, Toki F, Oyama H, Shimizu K, Shiratori K. Long term outcome of autoimmune pancreatitis after oral prednisolone therapy. Intern Med 2006; 45(8): 497-501.

[33] Taniguchi T, Seko S, Azuma K, et al. Autoimmune pancreatitis detected as a mass in the head of the pancreas with continguous fibrosis around the superior mesenteric artery. Dig Dis Sci 2001; 46: 187-191.

[34] Egawa N, Irie T, Tu Y, Kamisawa T. A case of autoimmune pancreatitis with initially negative autoantibodies turning positive during the clinical course. Dig Dis Sci 2003; 48: 1705-8.

[35] Ito T, Nakano I, Koyanagi S, et al. Autoimmune pancreatitis as a new clinical entity. Three cases of autoimmune pancreatitis with effective steroid therapy. Dig Dis Sci 1997; 42: 1458-68.

[36] Wada K, Shinoda T, Yoshimoto H. A case report of autoimmune pancreatitis with rapidly developing hyperglyemia and hypertension in a chronic hemodialysis patient. Ther Apher Dial 2007; 11: $150-4$.

[37] Tanaguchi T, Seko S, Azuma K, et al. Autoimmune pancreatitis detected as a mass in the tail of the pancreas. J Gastroenterol Hepatol 2000; 15 : 461-4.

[38] Chen R, Adams D. IgG4 levels in Non-Japanese patients with autoimmune sclerosing pancreatitis. N Engl J Med 2002; 346: 1919.

[39] Sahin P, Pozsar J, Simon K, Illyes G, Laszio F, Topa L. Autoimmune pancreatitis associated with immune-mediated inflammation of the papilla of Vater: report on two cases. Pancreas 2004; 29:1626. 